\title{
Unknown Object Detection by Punching: An Impacting-based Approach to Picking Novel Objects
}

\author{
Yusuke Maeda $^{1}$, Hideki Tsuruga ${ }^{2}$, Hiroyuki Honda ${ }^{2}$, and Shota Hirono ${ }^{2}$ \\ 1 Faculty of Engineering, Yokohama National University, 79-5 Tokiwadai, \\ Hodogaya-ku, Yokohama, 240-8501 JAPAN, \\ maeda@ynu.ac.jp, \\ WWW home page: http://www.iir.me.ynu.ac.jp/ \\ 2 Graduate School of Engineering, Yokohama National University
}

\begin{abstract}
In this paper, a method for unknown object detection based on impacting and keypoint tracking is presented. In this method, a robot perturbs object positions by punching the floor on which the objects are placed, to detect each of the objects individually from camera images before and after the punching. The detection method utilizes consistent movements of the keypoints of each object according to its rigid-body motion. After the detection, a grasp of each of the detected objects is planned based on extracting its two parallel edges. The proposed method is successfully applied to picking up of mahjong tiles by an industrial manipulator.
\end{abstract}

Keywords: Interactive perception, Segmentation, Picking

\section{Introduction}

Traditionally robots are used in structured environments to perform simple and repetitive operations. However, to perform complex and elaborate tasks like humans, robots have to understand non-structured environments. A straightforward solution to understand cluttered and non-structured scenes is to enhance the sensing ability of robots, but it has a limitation in nature: for example, it is impossible to distinguish between movable objects and immobile projections only from their appearance. Thus active sensing or interactive perception [3] to understand scenes through sensing and manipulation is important.

Metta and Fitzpatrick showed that simple operations of a robot such as poking and prodding bring better visual information to aid object recognition [12]. Chang et al. proposed adaptive pushing to singulate objects from a pile, which helps to improve picking success rates [4]. Hermans et al. proposed guided pushing to singulate objects on a table [9]. Gupta et al. presented spreading of piled objects to singulate them and aid object recognition for robotic sorting [6]. Katz et al. used pushing to singulate piled objects to clear them [10]. Schiebener et al. studied object segmentation using manipulation by a humanoid robot [13]. 


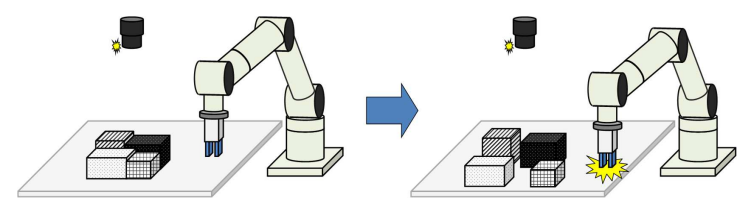

Fig. 1: Impacting for Detection of Unknown Objects

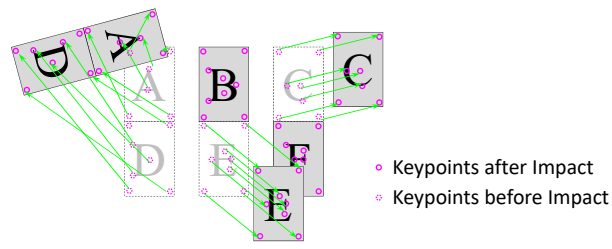

Fig. 2: Keypoint Tracking for Object Segmentation

In this method, unknown rigid objects in a cluttered environment are pushed by the robot and detected through analysis of motion of color-annotated point cloud obtained by stereo vision.

The previous studies demonstrated the usefulness of interactive perception by perturbing environments through robot operations. However, in these methods, perturbed region in a scene is limited because they use robot operations through direct contacts with objects, like pushing. Moreover, occlusion by robot bodies during operations can be a nasty problem for object detection. In this paper, we introduce a new approach to robotic interactive perception so that a robot can understand a broader region in a scene: impacting. By punching the floor on which the objects are located, objects in a large area can be perturbed without direct contacts between the robot and the objects (Fig. 1). Even novel objects can be detected through keypoint tracking between scene images before and after impacting (Fig. 2). By selecting appropriate punching points, occlusion can be avoided.

In this paper, for simplicity, we use a monocular camera to detect unknown objects on a floor. Some experiments on picking of them are shown. This paper is organized as follows: this section is introduction; our proposed method is overviewed in Section 2; its details are described in Section 3; some experimental results are shown in Section 4; Section 5 summarizes this paper.

\section{Overview of Impacting-Based Object Detection}

Our impacting-based object detection can be summarized as follows:

1. A scene image is obtained from a camera. Then a robot makes an impact to the scene to perturb the object positions: for example, punching a table on which objects are located. After the impacting, a new scene image is obtained from the camera. 
2. The images before and after impacting are analyzed. First, feature points in both of the images are extracted. Here we use SIFT keypoints [11], which are invariant to orientation changes. We also use Harris corners [8] to detect object corners mainly for textureless objects. Then we find one-to-one correspondences of the keypoints between the images before and after impacting, which stand for the movements of the keypoints by impacting.

3. The keypoints are grouped so that each group corresponds to an object. A RANSAC-based method is used to estimate the homogeneous transformation matrix that stands for the rigid-body motion of each object by impacting. We adopt $\alpha$-shape [5] to segment each object, which may be concave.

4. The robot picks up one of the detected objects. A grasp for the object is synthesized based on the segmentation result.

\section{Details of Object Detection}

\subsection{Keypoint Detection}

In this study, SIFT keypoints and Harris corners are detected for novel object detection. The former is used for textured objects and the latter is mainly for textureless objects. 128-dimensional SIFT descriptors are calculated not only for the SIFT keypoints but also for the Harris corners for keypoint tracking.

\subsection{Keypoint Matching}

We find keypoint correspondence between images before and after impacting. Euclidean distance between SIFT descriptors is adopted as the index for the correspondence. Additionally the positional difference between two keypoints is considered in finding the correspondence. Because keypoint movements by impacting should be small, we ignore keypoint pairs with large positional distance to omit incorrect correspondence even when there are two or more objects with identical textures. Additionally we ignore keypoint pairs with too small positional distance to omit background keypoints.

For each of the keypoints detected in the image before impacting, we calculate the Euclidean SIFT distances and the positional distances with all the keypoints detected in the image after impacting. The keypoint that has the minimum SIFT distance and the positional distance larger than a threshold $K_{\mathrm{bg}}$ and smaller than a threshold $K_{\text {dist }}$ is adopted as the correspondent, if any.

\subsection{Keypoint Pair Filtering}

The keypoint pairs found in the matching above are filtered so that the keypoint correspondences are one-to-one. If a keypoint in the image after impacting has multiple correspondents in the image before impacting, we keep only the pair with the minimum positional distance and remove the others (Fig. 3). 


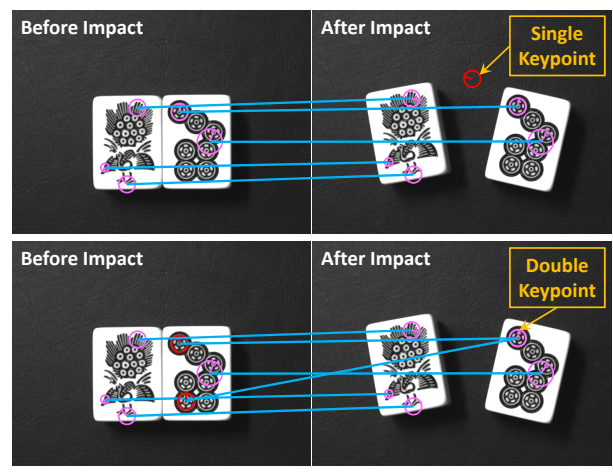

Fig. 3: Keypoint Pair Filtering

\subsection{Object Tracking}

We perform object tracking using RANSAC (Random Sampling Consensus) based on the result of keypoint tracking above as follows:

1. Two keypoint pairs are selected at random.

2. If the positional distance between the keypoints of the pairs in the image before impacting $d_{\text {bef }}$ is almost equal to that in the image after impacting $d_{\text {aft }}$, the two pairs can be a part of the rigid-body motion of an object. Concretely, if $\left\|d_{\text {bef }}-d_{\text {aft }}\right\|<K_{\text {len }}$, where $K_{\text {len }}$ is a threshold, we calculate the 2D homogeneous transformation matrix $\boldsymbol{H}$ that maps the keypoints of the two pairs in the image before impacting on those in the image after impacting.

3. If $\boldsymbol{H}$ is almost equal to a previously calculated one $\boldsymbol{H}^{\prime}, \boldsymbol{H}^{\prime}$ gets one vote. Concretely, if the difference of translation between $\boldsymbol{H}$ and $\boldsymbol{H}^{\prime}$ is smaller than a threshold $K_{\text {trans }}$ and the difference of rotation between $\boldsymbol{H}$ and $\boldsymbol{H}^{\prime}$ is smaller than a threshold $K_{\text {rot }}$, we regard $\boldsymbol{H}$ and $\boldsymbol{H}^{\prime}$ as identical. Otherwise, $\boldsymbol{H}$ gets one vote.

4. Repeat the above $N_{\text {vote }}$ times.

The homogeneous transformation matrices that get more than $K_{\text {vote }}$ votes are considered likely to correspond to the rigid-body motions of objects, where $K_{\text {vote }}$ is a threshold.

Then we group keypoint pairs for the elected homogeneous transformation matrices for object tracking. For each of the elected homogeneous transformation matrices, $\boldsymbol{H}^{*}$, we find all the keypoint pairs that match $\boldsymbol{H}^{*}$. If a keypoint pair $\left(\boldsymbol{p}_{\text {bef }}, \boldsymbol{p}_{\text {aft }}\right)$ satisfies

$$
\left\|\boldsymbol{H}^{*} \boldsymbol{p}_{\text {bef }}-\boldsymbol{p}_{\text {aft }}\right\|<K_{\text {diff }},
$$

where $K_{\text {diff }}$ is a threshold, we include it in the group of $\boldsymbol{H}^{*}$. All the grouped keypoint pairs stand for a tracked object. 
However, $\boldsymbol{H}^{*}$ could have a certain error because it is calculated only from two keypoint pairs in the beginning. Thus we recalculate the homogeneous transformation matrix $\boldsymbol{H}^{* *}$ from all the keypoint pairs that match $\boldsymbol{H}^{*}$ with the method by Arun et al. [2]. Then we group all the keypoint pairs that match $\boldsymbol{H}^{* *}$ such that

$$
\left\|\boldsymbol{H}^{* *} \boldsymbol{p}_{\text {bef }}-\boldsymbol{p}_{\text {aft }}\right\|<K_{\text {diff }}^{\prime},
$$

where $K_{\text {diff }}^{\prime}\left(<K_{\text {diff }}\right)$ is a threshold. All the keypoint pairs that match $\boldsymbol{H}^{* *}$ give us an improved result of object tracking.

\subsection{Object Segmentation}

We segment a tracked object for each of the keypoint pair groups in the above. For the keypoints in the image after impacting in a group of keypoint pairs, we calculate $\alpha$-shape [5], which can represent not only convex but also concave shapes. The minimum value of $\alpha$ such that all the keypoints in the group are included in the $\alpha$-shape is adopted. The $\alpha$-shape approximates the shape of the tracked object.

\subsection{Picking}

Here we select the $\alpha$-shape with the minimum area as the picking target. The minimum-area criterion is adopted to avoid selecting slightly moved background.

Then we consider how to grasp the $\alpha$-shape with a parallel gripper for picking. Harada et al. proposed a method to synthesize parallel gripper grasps by finding near-parallel surfaces of 3D objects [7]. In this study we simplify their method for its application to 2D objects (Fig. 4).

The grasp synthesis can be summarized as follows:

1. Consider three adjacent points in the $\alpha$-shape. If the three points are nearly in line, that is, the angle made by the three points is larger than a threshold $\theta_{\text {line }}$, the midpoint of the three is removed. Repeat this process to obtain a simplified $\alpha$-shape.

2. Find clusters of adjacent line segments that are located within two parallel lines with distance $h_{\max }$. Each of the found clusters is considered as a graspable region and approximated as a line segment.

3. Consider one of the obtained clusters in the descending order of the length of its approximated line segment. Then find its opposing cluster, if any. If there are multiple opposing clusters, the cluster closest to parallel is selected.

4. Synthesize a parallel grasp for the opposing clusters. The gripper is rotated to align with the two approximated line segments and translated to the center of the two approximated line segments. 


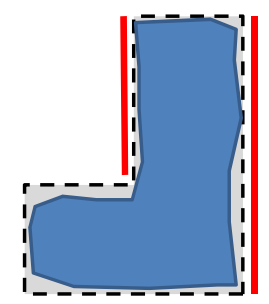

Fig. 4: Finding Near-Parallel Edges for Grasping

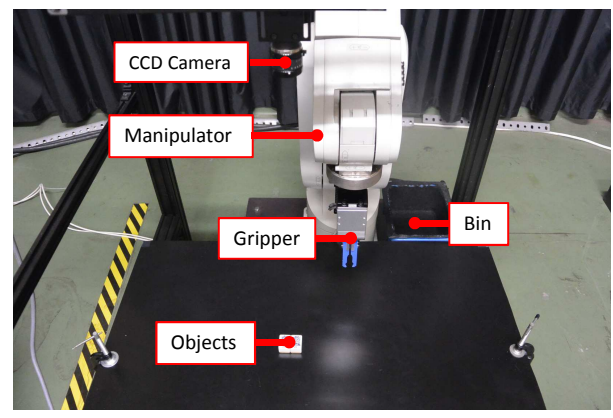

Fig. 5: Experimental Setup

\section{Experiments}

\subsection{Experimental Setup}

The experimental setup is shown in Fig. 5. A 6-axis manipulator (RV-1A by Mitsubishi Electric) equipped with a parallel gripper (ESG1-SS-2815 by Taiyo) is used. A spring mechanism is installed between the manipulator and the gripper to absorb shock in impacting. A grayscale camera (Flea2 FL2G-13S2M by Point Grey Research, $1296 \times 964$ pixels) is placed above the manipulator. A Linux PC with Intel Core i7-3770K $(3.50 \mathrm{GHz})$ is used to control the manipulator and the camera. OpenCV [1] is used for image processing implementation.

Mahjong tiles are used as objects to be picked up because they have a wide variety in texture, from textureless to highly textured. The size of each of the mahjong tiles is $19.4[\mathrm{~mm}]($ width $) \times 26.5[\mathrm{~mm}]($ depth $) \times 16.1[\mathrm{~mm}]$ (height) Its weight is about 14 [gf].

\subsection{Assumptions}

We set an impacting point on the floor, to be punched by moving down the gripper of the manipulator vertically. The floor is sloped about five degrees to make object movement by impacting easier. The position of the floor is known.

All the objects are placed on the floor. Thus picking is possible if the objects are localized on the floor with the camera calibrated in advance.

Parameters used in the experiments are shown in Table 1. 
Table 1: Method Parameters

\begin{tabular}{|l|l|}
\hline$K_{\text {dist }}$ & 20 [pixel] \\
\hline$K_{\text {bg }}$ & 2 [pixel] \\
\hline$K_{\text {len }}$ & 2 [pixel] \\
\hline$K_{\text {trans }}$ & 4 [pixel] \\
\hline$K_{\text {rot }}$ & $10[\mathrm{deg}]$ \\
\hline$K_{\text {diff }}$ & $2[$ pixel] \\
\hline$K_{\text {diff }}^{\prime}$ & $1[$ pixel] \\
\hline$N_{\text {vote }}$ & 1000 \\
\hline$K_{\text {vote }}$ & 100 \\
\hline$\theta_{\text {line }}$ & $170[\mathrm{deg}]$ \\
\hline$h_{\text {max }}$ & $3[\mathrm{~mm}]$ \\
\hline
\end{tabular}

Table 2: Result of Detection Experiments

\begin{tabular}{|c|c|}
\hline Successful & $14 / 18$ \\
\hline Unsuccessful & $4 / 18$ \\
\hline Success Rate & $78 \%$ \\
\hline
\end{tabular}

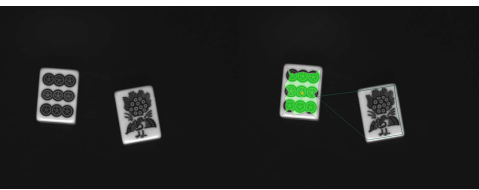

(a) Example 1

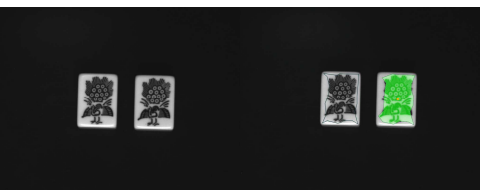

(c) Example 3

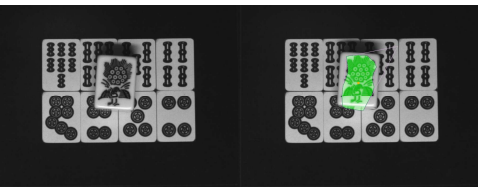

(e) Example 5

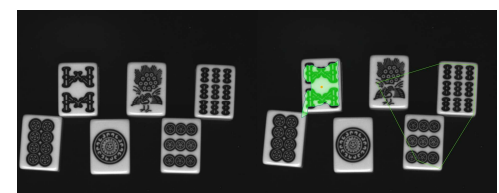

(b) Example 2

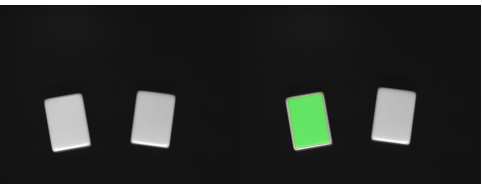

(d) Example 4

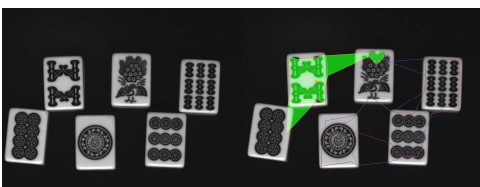

(f) Example 6

Fig. 6: Experiments of Object Detection

\subsection{Experiments of Object Detection}

Here we show the experimental results of object detection. Our proposed method is applied to eighteen sample image pairs before and after impacting.

Results are shown in Table 2 and Fig. 6. The success/failure of detection shown in Table 2 is determined subjectively according to the difference between the outer shape of the target object and its $\alpha$-shape segmentation in terms of picking feasibility. 


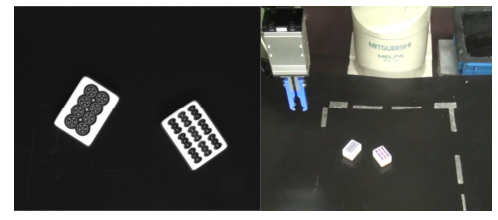

(a) before 1st impacting

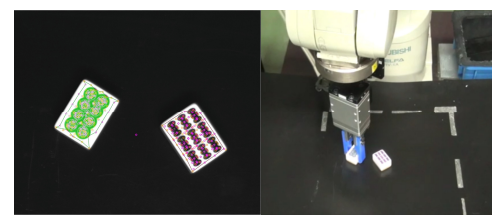

(c) 1st segmented object

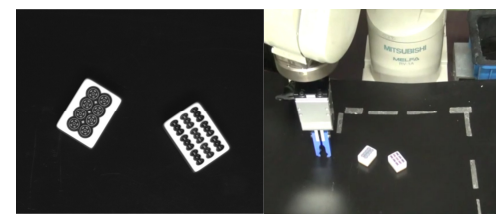

(b) after 1st impacting

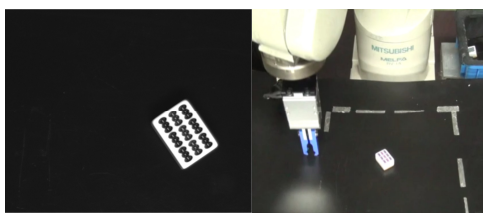

(d) after 2nd impacting

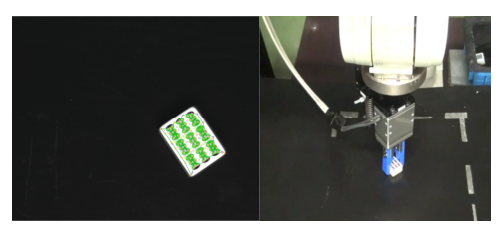

(e) 2nd segmented object

Fig. 7: Picking Differently Textured Objects

Fig. 6 shows some examples of the segmentation results. The left and right are images before and after impacting, respectively. Segmented objects are specified by green regions. Fig. 6a includes two differently textured objects; Fig. 6b and Fig. 6f include six differently textured objects; Fig. 6c includes two identically textured objects; Fig. 6d includes two textureless objects; Fig. 6e includes one textured object placed on the photo of mahjong tiles (one genuine object on background fake objects). Fig. 6a-Fig. 6e show successful object segmentation.

On the other hand, Fig. 6f shows unsuccessful object segmentation, which includes not only the upper-left target object but also outliers on the neighbor objects. The outliers are keypoints that moved consistently with the movement of the target object and it is difficult to omit them when using only one image after impacting. Thus to solve this problem, calculating intersection of segmented regions using multiple frames after impacting would be useful.

\subsection{Picking Experiments}

We performed picking experiments with our proposed method. Success of picking is defined as transportation of the picked object to a goal bin. Experiments are terminated when no object movements by impacting are found. 


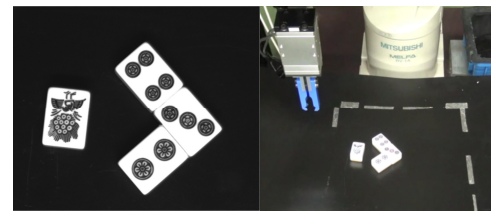

(a) before 1st impacting

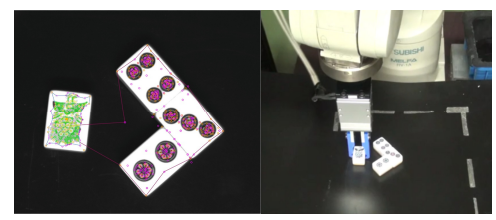

(c) 1st segmented object

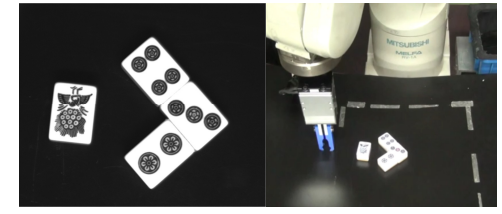

(b) after 1st impacting

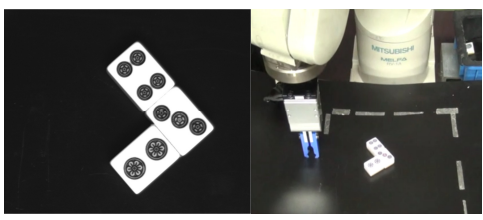

(d) after 2nd impacting

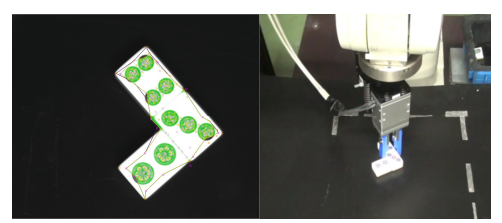

(e) 2nd segmented object

Fig. 8: Picking including L-shaped Object

Differently Textured Objects Here we show a result of picking two differently textured objects. Some scenes in this experiment are shown in Fig. 7. The left halves of the subfigures are photos captured by the camera for image processing. Segmented objects are specified by interposed green regions. The two objects were successfully picked up after impacting twice in total.

L-shaped Object Here we show a result of picking objects including an Lshaped one, which is composed of three connected mahjong tiles. Some scenes in this experiment are shown in Fig. 8. Two faces of the L-shaped object were grasped and its picking was successful.

Picking with a Textured Background Here we show a result of picking an object placed on a textured background. Some scenes in this experiment are shown in Fig. 9. In this case, the object placed on eight fake objects was found correctly and picking was successful.

\section{Conclusion}

This paper presented a method to detect novel objects by impacting. In contrast to most of previous studies on interactive perception through pushing, the 


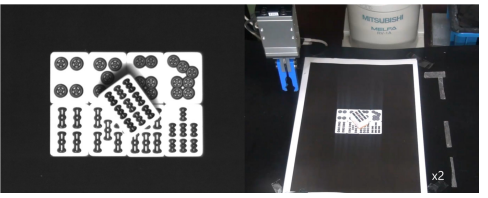

(a) before impacting

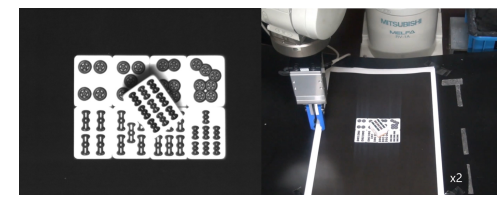

(b) after impacting

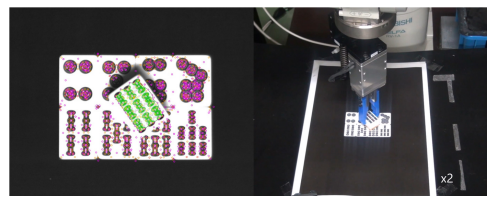

(c) segmented object

Fig. 9: Picking with a Textured Background

method can detect objects in wider region and suffers less from occlusion because impacting does not need direct contacts with the objects. We showed that it was possible to detect novel objects including textured and textureless mahjong tiles in experiments. Moreover, by grasp synthesis based on object segmentation, we showed robotic picking of the detected novel objects in experiments.

The success rate of object detection should be improved in future work. Using three or more frames in keypoint tracking would be useful. Grasp synthesis should also be improved for more reliable picking.

Currently, our method is applicable only to $2 \mathrm{D}$ objects. Its extension to $3 \mathrm{D}$ objects should also be addressed. It would be possible through 2D keypoint tracking with stereo vision, or 3D keypoint tracking with depth sensing.

\section{References}

1. OpenCV. http://opencv.org/

2. Arun, K.S., Huang, T.S., Blostein, S.D.: Least-squares fitting of two 3-D point sets. IEEE Trans. on Pattern Analysis and Machine Intelligence 9(5), 698-700 (1987)

3. Bohg, J., Hausman, K., Sankaran, B., Brock, O., Kragic, D., Schaal, S., Sukhatme, G.S.: Interactive perception: Leveraging action in perception and perception in action. IEEE Trans. on Robotics 33(6), 1273-1291 (2017)

4. Chang, L., Smith, J.R., Fox, D.: Interactive singulation of objects from a pile. In: Proc. of 2012 IEEE Int. Conf. on Robotics and Automation. pp. 3875-3882 (2012)

5. Edelsbrunner, H., Kirkpatrick, D., Seidel, R.: On the shape of a set of points in the plane. IEEE Trans. on Information Theory 29(4), 551-559 (1983)

6. Gupta, M., Müller, J., Sukhatme, G.S.: Using manipulation primitives for object sorting in cluttered environments. IEEE Trans. on Automation Science and Engineering 12(2), 608-614 (2015)

7. Harada, K., Tsuji, T., Nagata, K., Yamanobe, N., Maruyama, K., Nakamura, A., Kawai, Y.: Grasp planning for parallel grippers with flexibility on its grasping 
surface. In: Proc. of IEEE Int. Conf. on Robotics and Biomimetics. pp. 1540-1546 (2011)

8. Harris, C., Stephens, M.: A combined corner and edge detector. In: Proc. of Fourth Alvey Vision Conf. pp. 147-151 (1988)

9. Hermans, T., Rehg, J.M., Bobick, A.: Guided pushing for object singulation. In: Proc. of 2012 IEEE/RSJ Int. Conf. on Intelligent Robots and Systems. pp. 4783$4790(2012)$

10. Katz, D., Venkatraman, A., Kazemi, M., Bagnell, J.A., Stentz, A.: Perceiving, learning, and exploiting object affordances for autonomous pile manipulation. Autonomous Robots 37(4), 369-382 (2014)

11. Lowe, D.G.: Object recognition from local scale invariant features. In: Proc. of 1999 IEEE Int. Conf. on Computer Vision. pp. 1150-1157 (1999)

12. Metta, G., Fitzpatrick, P.: Better vision through manipulation. Adaptive Behavior 11(2), 109-128 (2003)

13. Schiebener, D., Ude, A., Asfour, T.: Physical interaction for segmentation of unknown textured and non-textured rigid objects. In: Proc. of 2014 IEEE Int. Conf. on Robotics and Automation. pp. 4959-4966 (2014) 